Fundación

Miguel Lillo

Tucumán

Argentina

\title{
Psilocybe cubensis (Agaricales, Basidiomycota) en Ecuador
}

Psilocybe cubensis (Agaricales, Basidiomycota) in Ecuador

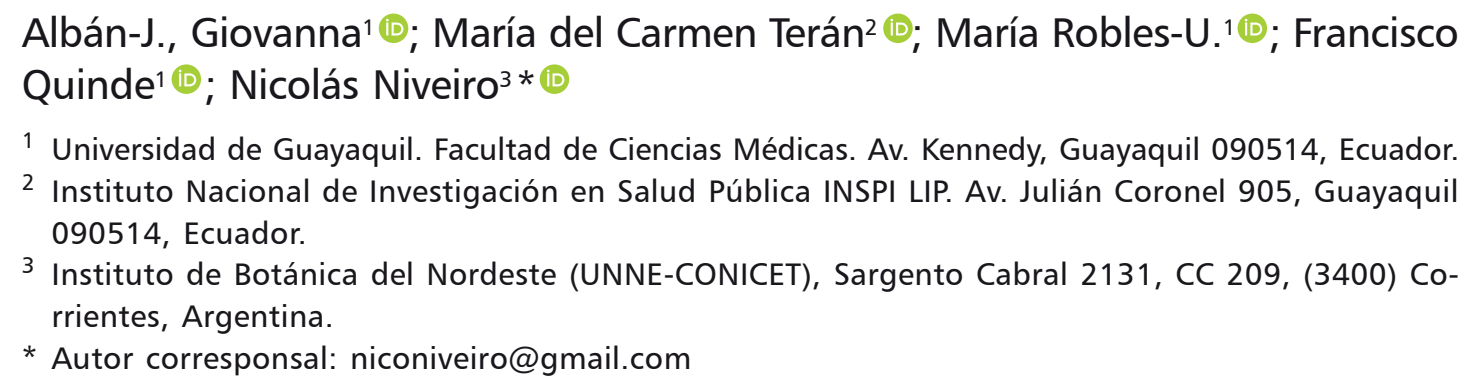

\section{RESUMEN}

Psilocybe cubensis es una especie con propiedades alucinógenas utilizada tradicionalmente por diversas comunidades originarias, pero más recientemente, utilizada con fines recreativos, a pesar de su ilegalidad. Es una especie coprófila que fructifica en el estiércol vacuno de las praderas de áreas tropicales y subtropicales de América, Asia y Oceanía. En el presente estudio se registra $P$. cubensis por primera vez para los pastizales de altura de Ecuador. Además, se ofrece una descripción detallada (macro y microscópica) y se discuten las diferencias con especies similares.

Palabras clave - Agaricomycetes; Psilocybe subcubensis; Strophariaceae.

\begin{abstract}
Psilocybe cubensis is a species with hallucinogenic properties traditionally used by various native communities, but more recently, it has been used for recreational purposes, despite its illegality. This is a coprophilous species bearing fruits on the cow dung in the grasslands of tropical and subtropical areas of America, Asia and Oceania. In the present study, P. cubensis is recorded for the first time in the highland grasslands of Ecuador. In addition, a detailed description (macro and microscopical) is provided and differences with similar species are discussed.

Keywords - Agaricomycetes; Psilocybe subcubensis; Strophariaceae.

Ref. bibliográfica: EAlbán-J., G.; Terán, M. del C.; Robles-U, M.; Quinde, F.; Niveiro, N. 2021. Psilocybe cubensis (Agaricales, Basidiomycota) en Ecuador. Lilloa 58 (2): 86-94. doi: https://doi.org/10.30550/j.lil/202 1.58.1/2021.09.30

> Recibido: 5 de abril 2021 - Aceptado: 30 de septiembre 2021 - Publicado en línea: 25 de octubre 2021.

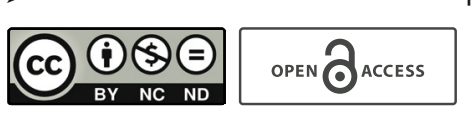

> URL de la revista: http://lilloa.lillo.org.ar

- Esta obra está bajo una Licencia Creative Commons Atribución - No

Comercial - Sin Obra Derivada 4.0 Internacional.
\end{abstract}




\section{INTRODUCCIÓN}

Desde la época prehispánica, diversos pueblos Mesoamericanos utilizaron hongos con propiedades alucinógenas tanto en ceremonias y rituales sagrados como con fines medicinales (Wasson, 1957; Heim, 1957, 1959; Heim y Wasson, 1958; Guzmán, 1959; 2016), sin embargo, actualmente son ampliamente utilizadas con fines recreativos (Guzmán, 2003; Van Amsterdam et al., 2011).

El género Psilocybe fue escasamente muestreado en Ecuador, Guzmán (1978) describe cuatro especies: P. alnetorum (Singer) Singer, P. coprophila (Bull.) Kumm. [= Deconica coprophila (Bull.) P. Karst.], P. panaeoliformis Murrill, y P. subcubensis Guzmán. Casi simultáneamente, Singer $(1975,1978)$ presenta dos trabajos en donde describe varias especies de Agaricales para Ecuador, en donde incluye solo a $P$. aequatoriae Singer (Singer, 1978), especie posteriormente transferida como Deconica aequatoriae (Singer) Ram.-Cruz \& Guzmán (Ramírez Cruz et al., 2012). Posteriormente se describen P. zapotecorum Heim. y P. yungensis Singer \& A.H. Sm. (Guzmán, 1983; Coimbra, 2015), y Læssøe y Petersen (2008) registran a P. cubensis (Earle) Singer y P. plutonia (Berk. \& M. A. Curtis) Sacc. para Ecuador.

Psilocybe cubensis y $P$. subcubensis son especies coprófilas que fructifican en estiércol vacuno en praderas de zonas tropicales y subtropicales (Singer, 1961; Piepenbring, 2008; Guzmán et al. 2012). Son las especies más utilizadas para su consumo, debido a que presentan un mayor rango de distribución y están asociadas a ambientes disturbados, con ganado, por lo que generalmente se encuentran en cercanías de centros urbanos (Guzmán, 1983). Læssøe y Petersen (2008) registraron a la primera en campos de pastoreo de las zonas subtropicales de Ecuador, pero sin una descripción microscópica detallada. Considerando que ambas especies son indistinguibles macroscópicamente, el presente trabajo tiene como objetivo ofrecer una descripción macro y micromorfológica detallada de $P$. cubensis y discutir los caracteres distintivos entre las especies relacionadas.

\section{MATERIALES Y MÉTODOS}

Los ejemplares fueron colectados de forma aleatoria en la parroquia La Esperanza, ubicada en las cercanías de la localidad de Ibarra, provincia de Imbabura, Ecuador (004'52” S 78²6’38” W). Los pisos altitudinales del cantón varían entre 1730 y $4300 \mathrm{~m}$ snm, desde la zona de páramo correspondiente al piso altitudinal del bosque húmedo montano, extendiéndose por un relieve escarpado y una topografía irregular hasta llegar a un valle de características xerofíticas correspondientes al bosque seco montano bajo (Valencia-P y Garzón-C, 2018). Presenta una temperatura media de $15.9^{\circ} \mathrm{C}$ con mínimas de $4^{\circ} \mathrm{C}$. El análisis hidrometeorológico determina que la variación de la precipitación determina épocas de sequía entre los meses de junio y septiembre, y épocas lluviosas en los meses de marzo a mayo y de octubre a noviembre (Valencia-P y Garzón-C, 2018).

Los ejemplares fueron fotografiados y descritos macroscópicamente. Para la identificación taxonómica se analizaron macro y microscópicamente los ejemplares siguiendo los criterios y terminología propuesta por Vellinga (1988) y Lodge 
et al. (2004). Para el análisis microscópico se realizaron cortes a mano alzada y se analizaron: elementos del revestimiento piléico, del contexto de píleo y pie, de las laminillas, esporas, basidios y cistidios. Los mismos fueron montados en $\mathrm{KOH} 5 \%$ y teñidos con floxina acuosa al 1\%, y reactivo de Melzer (Wright y Albertó, 2002). Las medidas de todas las estructuras microscópicas se realizaron directamente con objetivo de inmersión a 1000X o bien, mediante fotografías tomadas del microscopio con cámara incorporada Leica EC3 utilizando el software ImageJ (Schneider et al., 2012). Para las distintas estructuras microscópicas, se proporcionan los intervalos del valor mínimo - máximo, separados con un guion. Para las esporas se brindan los rangos de medidas de la longitud, diámetro de la cara frontal y lateral, el valor promedio $(x)$, el coeficiente Q (longitud/diámetro), el valor medio del coeficiente Q ( Qx) y el número de esporas medidas (n). La identificación se realizó según los caracteres morfológicos utilizando las claves de identificación de Niveiro et al. (2014) para nivel genérico, y Guzmán (1983) para las especies. Los autores de los nombres científicos se indican de acuerdo al Index Fungorum - Authors of Fungal Names (2021) y las siglas de los herbarios según Thiers (2021). El material colectado fue procesado y posteriormente depositado como referencia en la colección micológica del Instituto de Botánica del Nordeste (CTES).

\section{RESULTADOS Y DISCUSIÓN}

Psilocybe cubensis (Earle) Singer, Sydowia 2: 37 (1948).

三Stropharia cubesis Earle, Inf. an. Estac. Cent. agr. Cuba 1: 240 (1906). Tipo: Cuba, Santiago de las Vegas, Earle 109 (NY00764563). Imagen digital disponible en http://sweetgum.nybg.org/science/vh/specimen-details/?irn=509066. Figs. 1-3.

Píleo 20-80 mm diám., parabólico a convexo cuando joven, volviéndose plano convexo con el centro umbonado en la madurez, superficie víscida, volviéndose seca, glabra, uniformemente castaño anaranjado (6A6-6C6) cuando joven, aclarándose hacia los márgenes en la madurez, de castaño anaranjado (6A6-6C6) en el centro, pasando a anaranjado claro (5A4), amarillo claro (4a4), blanco amarillento (1A2) hasta blanco (1A1). Laminillas anexas o adnatas, próximas, ventricosas, anchas, de hasta $4 \mathrm{~mm}$, grisácea violeta (13C2) cuando jóvenes, volviéndose marrón (10F6-8) a negra con tonalidades violáceas (10F-3, "greyish brown” o más oscuro incluso), generalmente moteadas, margen finamente fimbriado, blanquecino. Estípite 40-100 $\times$ 3-6 mm, central, cilíndrico ensanchándose hacia la base, base subbulbosa de hasta $10 \mathrm{~mm}$ diám., hueco, superficie blanco-cremoso (5A2 "orange White") o amarilloamarronado (5A3 "pale orange"), glabro a finamente fibrillosa, cuando se lesiona, adopta un color azul-verdoso, usualmente negro en materiales deshidratados. Anillo membranáceo con margen floculoso, supero, blanco, tornándose negruzco por el depósito de esporas, persistente pero muy frágil. Contexto carnoso, hasta $10 \mathrm{~mm}$ de espesor, blanquecino, tornándose azul-verdoso al corte. Esporada negra violácea.

Esporas $12-15 \times 8-10 \times(7,5) 8-9 \mu \mathrm{m}, x=13.5 \times 8.8 \times 8,5 \mu \mathrm{m}, \mathrm{Q}=1,40-1,80$, $\mathrm{Q} x=1,5, \mathrm{n}=25$; cara frontal subhexagonal, elipsoidales en vista lateral, superficie 


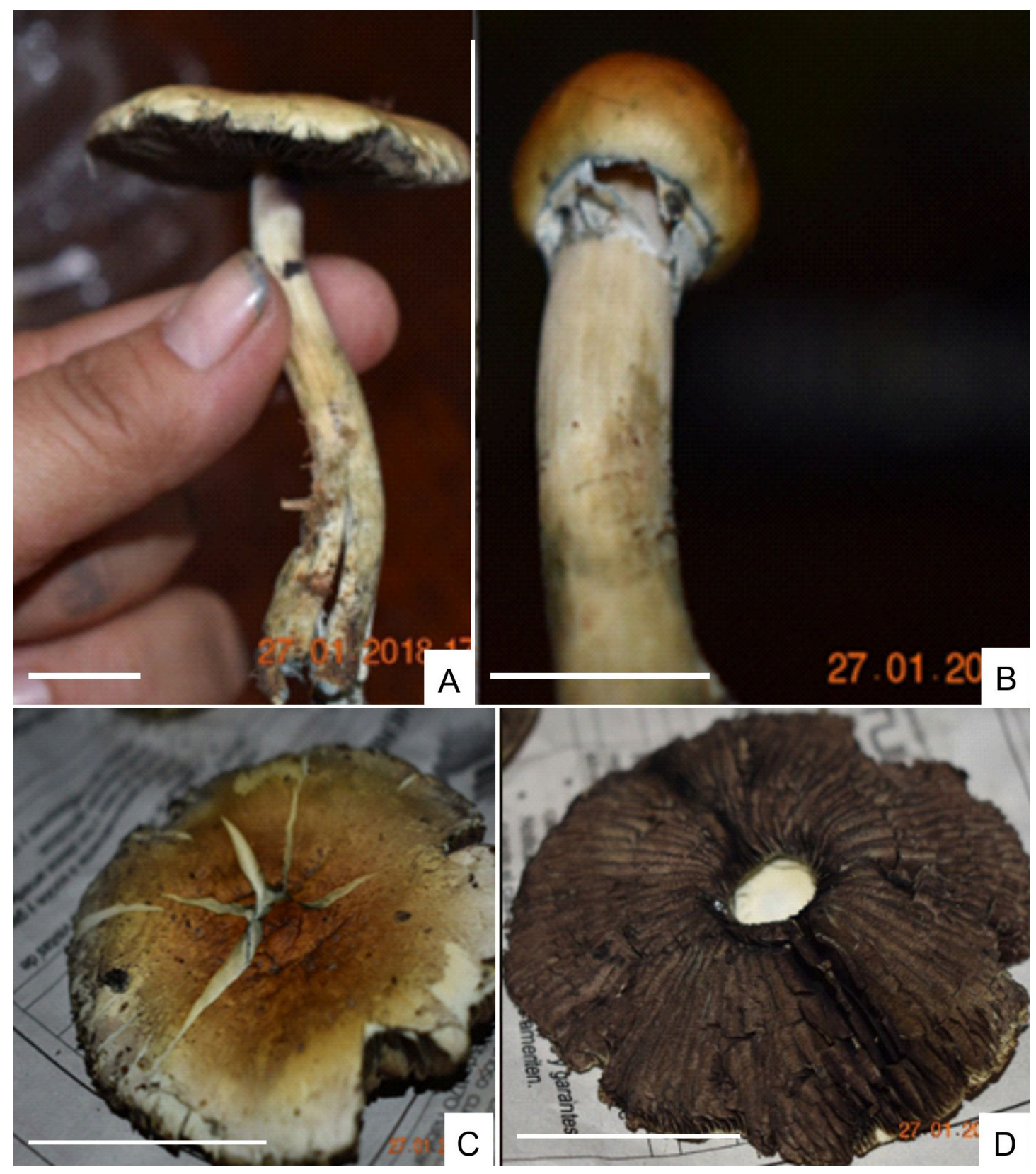

Fig. 1. Basidiomas de $P$ c cubensis. A) Basidioma maduro. B) Especimen joven. C) Detalle de la superficie del pileo. D) Detalle de las laminillas. Albán et al. s/n (CTES). Escala: 20 mm.

Fig. 1. Basidiomata of $P$. cubensis. A) Mature basidiome. B) Young basidiome. C) Detail of the pilear surface. D) Detail of the lamellae. Albán et al. s / n (CTES). Scale: 20 mm.

lisa, de paredes gruesas (hasta $1 \mu \mathrm{m}$ espesor), amarillentas amarronadas, con ápice truncado con poro germinativo. Basidios 23-29 $\times 9-10 \mu \mathrm{m}$, utriniformes a cilíndricos, de pared delgada, 4-esterigmados. Pleurocistidios (14) 19-25 × 7,5-11,5 $\mu \mathrm{m}$, ventricosos a subpiriformes, con ápice mucronado, de paredes delgadas, dispersos. Queilocistidios 16-22 × 7-10 $\mu \mathrm{m}$, lageniformes, ventricosos a anchamente fusiformes, de paredes delgadas, abundantes, formando una capa estéril en el margen de las laminillas. Trama himenoforal paralela, formada por hifas elongadas de hasta $7 \mu \mathrm{m}$ diám., hialinas. Subhimenio subcelular, formando una capa de hasta $20 \mu \mathrm{m}$ de espesor, con 


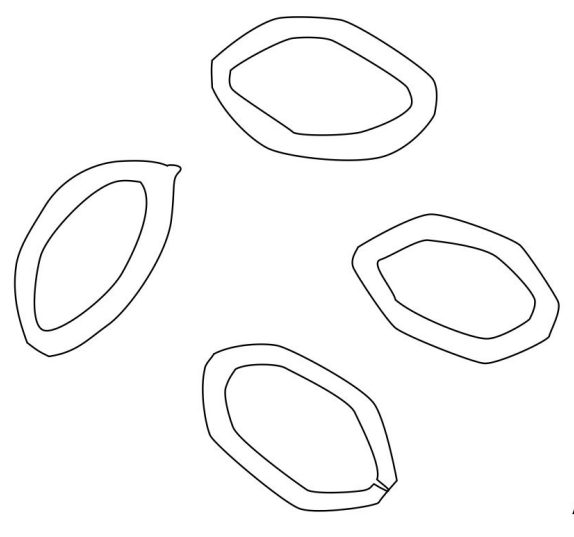

A
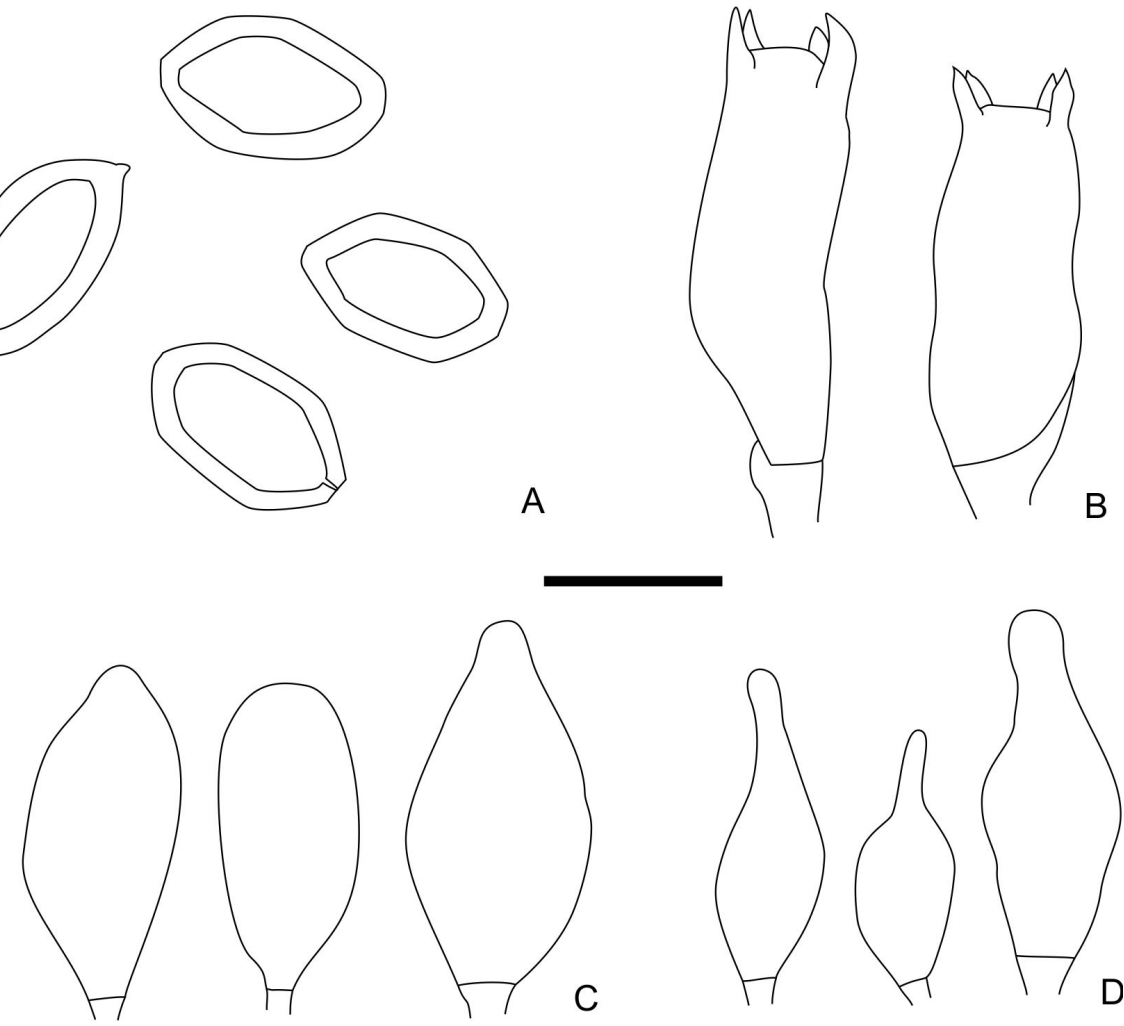

Fig. 2. Microscopia de P. cubensis. A) Esporas. B) Basidios. C) Pleurocistidios. D) Queilocistidios. Albán et al. s/n (CTES). Escala: $10 \mu \mathrm{m}$.

Fig. 2. Microscopy of $P$. cubensis. A) Basidiospores. B) Basidia. C) Pleurocystidia. D) Cheilocystidia. Albán et al. s/n (CTES). Scale: $10 \mu \mathrm{m}$.

células isodiamétricas de 9-12 $\mu \mathrm{m}$ diám. Pileipellis formado por un ixocutis de 90$170 \mu \mathrm{m}$ de espesor, formado por hifas subparalelas, de 1,5-3 $\mu \mathrm{m}$ de diám., hialinas, de paredes delgadas, orientadas radialmente, embebidas en una matriz gelatinosa. Subpellis formada por hifas de 4-7 $\mu \mathrm{m}$ diám., de paredes delgadas, con pigmentos castaños claros. Fíbulas presentes.

Hábitat.- Basidiomas gregarios, coprófilos, sobre excremento de animales vacunos.

Distribución.- Pantropical, se conoce en América, desde Estados Unidos hasta Argentina (Estados Unidos, México, Belice, Guatemala, Costa Rica, El Salvador, Panamá, Cuba, Dominica, Republica Dominicana, Puerto Rico, Martinica, Guadalupe, Trinidad y Tobago, Colombia, Venezuela, Guyana Francesa, Ecuador, Perú, Bolivia, Brasil, Argentina), en el sudeste de Asia (Camboya, Filipinas, India, Tailandia y Vietnam), y en Oceanía (Australia, Tasmania, Nueva Zelanda) (Guzmán, 1983, 2016; Guzmán et al., 1988, 2012; Piepenbring, 2008; Ramírez Cruz et al., 2013; Coimbra 2015).

Material examinado. - ECUADOR. Imbabura, Ibarra, Parroquia La Esperanza. Quebrada Arteza Huaicu, 0¹7’02,1” N 7805’27,3” W, 1835 m snm, 27-I-2018, G. Albán et al. s/n (CTES). 

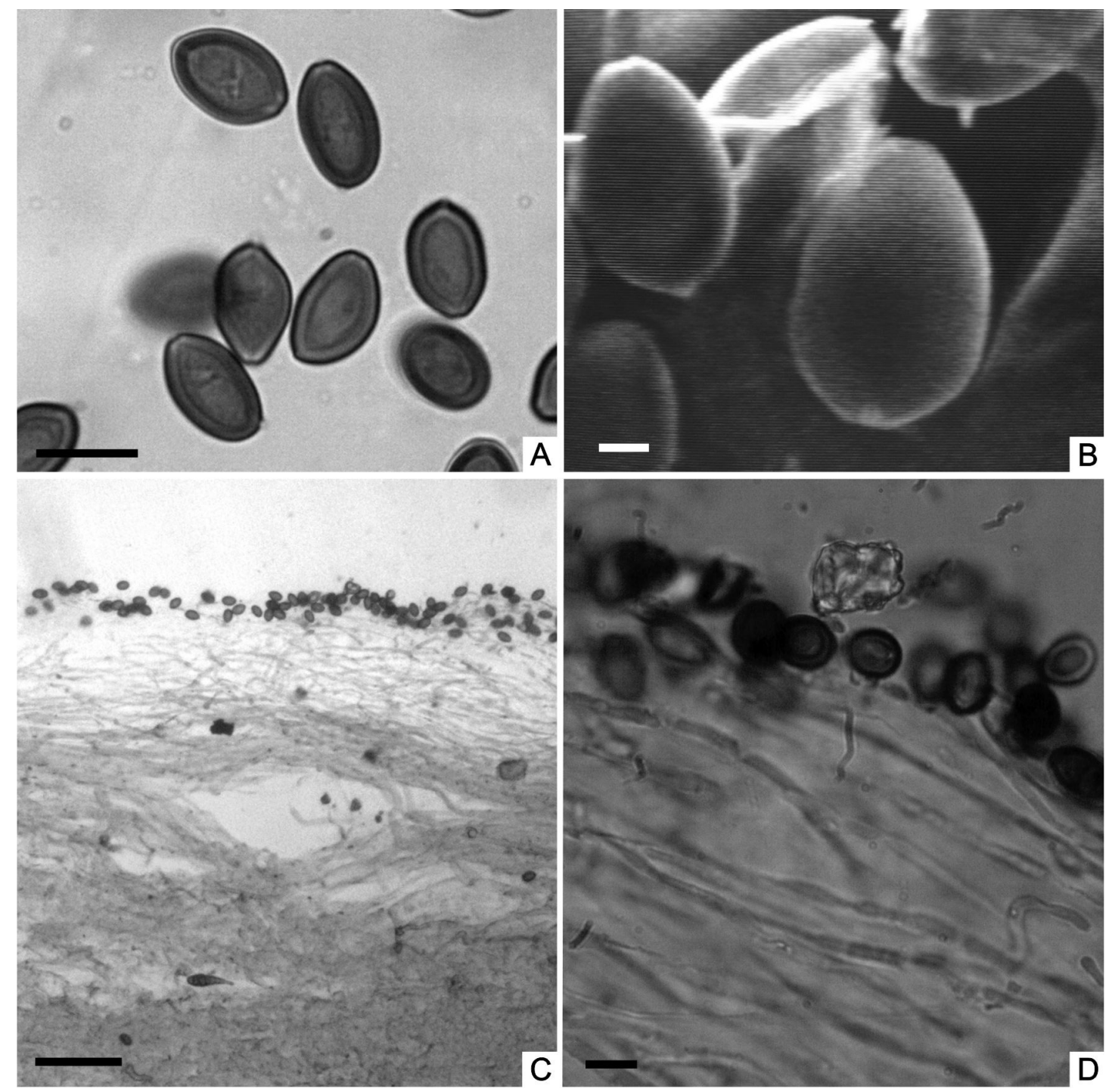

Fig. 3. Microscopia de P. cubensis. A) Esporas en microscopio óptico. B) Esporas en MEB. C) Pileipellis. D) Detalle de las hifas de la pileipellis. Albán et al. $\mathrm{s} / \mathrm{n}$ (CTES). Escala: $\mathrm{a}=10 \mu \mathrm{m}, \mathrm{b}=2 \mu \mathrm{m}, \mathrm{c}$ $=100 \mu \mathrm{m}, \mathrm{d}=10 \mu \mathrm{m}$.

Fig. 3. Microscopy of $P$. cubensis. A) Basidiospores under light microscope. B) Basidiospores at SEM. C) Pileipellis. D) Detail of the pileipellis hyphae. Albán et al. s/n (CTES). Scale: $a=10 \mu \mathrm{m}, \mathrm{b}=2 \mu \mathrm{m}$, $\mathrm{c}=100 \mu \mathrm{m}, \mathrm{d}=10 \mu \mathrm{m}$.

Observaciones. - P. cubensis forma parte de la Sección Cubensae (Guzmán, 1983) o para otros autores, de la sección Caerulescentes, tribu Cubensis (Singer y Smith, 1958; Singer, 1986). Esta especie se caracteriza por sus basidiomas de gran tamaño, su píleo blanquecino con el centro ocráceo, estípite con anillo membranáceo bien desarrollado, su hábito coprófilo, creciendo sobre excremento de ganado vacuno o equino, y por el cambio de color que presenta al tacto, volviéndose verdoso-azulado (Singer y Smith, 1958; Guzmán, 1983).

Por estas características macroscópicas, no es diferenciable de las especies más relacionadas, $P$. subcubensis y $P$. aquamarina (Pegler) Guzmán, las cuales se diferencian por sus esporas de menor tamaño, 11-13 (14) $\times 7,7-8,8 \mu \mathrm{m}$ en P. subcubensis (Guzmán, 
1983) y 10-11 (12) $\mu \mathrm{m}$ en P. aquamarina (Pegler, 1977 como Stropharia aquamarina; Guzmán et al., 2014). Es interesante destacar, que por el hábito coprófilo de $P$. cubensis, se cree que se introdujo a América por los españoles junto al ganado traído desde África, sin embargo, esta especie no es conocida para este último continente (Guzmán, 1983; Guzmán et al., 2014). No existen estudios adicionales, como por ejemplo de compatibilidad sexual o de relaciones filogenéticas, que permitan dilucidar si estas tres especies son independientes entre sí. Borovièka et al. $(2011,2015)$ y Ramírez Cruz et al. (2013) realizaron estudios filogenéticos en diferentes especies de Psilocybe, sin embargo, ninguno de estos estudios se centró sobre las relaciones de éstas especies. Ramírez Cruz et al. (2013) muestra que P. cubensis y P. subcubensis conforman un mismo clado bien soportado, pero solo en base a tres especímenes, por esta razón, nuevos estudios son necesarios para comprender las relaciones filogenéticas entre estas especies.

Una especie íntimamente relacionada, morfológica y filogenéticamente, es $P$. chuxiongensis Ma T. Ma \& K. D. Hyde, pero se diferencia por la ausencia de anillo, y el píleo convexo, no papilado (Ma et al., 2014). Otras dos especies descriptas para el sudeste asiático $P$. aerugineomaculans (Höhnel) Singer y $P$. subaeruginascens Höhnel (= Stropharia caerulescens S. Imai), se asemejan por la presencia de un estípite con anillo membranáceo, pero se diferencian por sus esporas de menor tamaño $(8,5-10 \times 7-8 \times$ 5,5-6,5 $\mu \mathrm{m}$ en $P$. aerugineomaculans) y 7,7-10 $\times$ 7-7,8 $\times 6-7 \mu \mathrm{m}$ en $P$. subaeruginascens) y habito cortícola en el primer caso (Singer y Smith, 1958).

Si bien $P$. cubensis fue anteriormente citado para Ecuador por Læssøe y Petersen (2008), en el presente trabajo se describe e ilustra macro y microscópicamente detalladamente a esta especie, corroborando la presencia de la misma mediante su análisis microscópico, y brindando los caracteres morfológicos necesarios para la realización de análisis taxonómicos comparativos con especímenes de otras regiones del mundo.

\section{AGRADECIMIENTOS}

El documento es producto del proyecto de Investigación de Fondos Concursables (FCI-017-2017) de la Universidad de Guayaquil, aplicado por medio de la Facultad de Ciencias Médicas que incluyó la participación de estudiantes de la carrera de medicina con el propósito de despertar el deseo de investigar y fortalecer sus conocimientos en Micología, esto permitió realizar dos estudios de campo, taxonomía del género Psilocybe y efectos psicotrópicos debido al consumo de especies alucinógenas. Las imágenes de microscopio electrónico de barrido se obtuvieron por cortesía de Dr. Yasuji Amano y Dra. Linda Díaz, del Instituto Nacional de Investigación en Salud Pública.

\section{BIBLIOGRAFÍA}

Borovièka, J., Noordeloos, M. E., Gryndler, M., Oborník, M. (2011). Molecular phylogeny of Psilocybe cyanescens complex in Europe, with reference to the position 
of the secotioid Weraroa novae-zelandiae. Mycological Progress 10 (2): 149-155. https://doi.org/10.1007/s11557-010-0684-3

Borovièka, J., Oborník, M., Støíbrný, J., E. Noordeloos, M., Parra-S, L. A., Gryndler M. (2015). Phylogenetic and chemical studies in the potential psychotropic species complex of Psilocybe atrobrunnea with taxonomic and nomenclatural notes. Peersonia 34: 1-9. https://doi.org/10.3767/003158515X685283

Coimbra, V. R. M. (2015). Checklist of Centran and South American Agaricales (Basidiomycota) II: Strophariaceae. Mycosphere 6 (4): 441-458. https://doi.org/10.5943/ mycosphere/6/4/6

Guzmán, G. (1959). Sinopsis de los conocimientos sobre los hongos alucinógenos mexicanos. Boletín de la Sociedad Botanica de México 24: 14-34.

Guzmán, G. (1978). The species of Psilocybe known from Central and South America. Mycotaxon 7: 225-255.

Guzmán, G. (1983). The genus Psilocybe. Beih. Nova Hedwigia 74: 1-439.

Guzmán, G. (2003). Traditional uses and abuses of hallucinogenic fungi: problems and solutions. International Fournal of Medicinal Mushrooms 5: 57-59. https://doi. org/10.1615/IntJMedMushr.v5.i1.80

Guzmán, G. (2016). Las relaciones de los hongos sagrados con el hombre a través del tiempo. Anales de Antropología 50 (1): 134-147. https://doi.org/10.1016/ j.antro.2015.10.005

Guzmán, G., Montoya-B, L., Bandala-M, V. M. (1988). Nuevos registros de los hongos alucinógenos del género Psilocybe en México y análisis de la distribución de las especies conocidas. Scientia Fungorum 3 (4): 255-65

Guzmán, G., Ramírez-G, F., Hyde, K. D., Karunarathna S. C. (2012). Psilocybe s.s. in Thailand: four new species and a review of previously recorded species. Mycotaxon 119: 65-81. https://doi.org/10.5248/119.65

Guzmán, G., Nixon, S. C., Ramírez-G, F., Cortés-P, A. (2014). Psilocybe s. str. (Agaricales, Strophariaceae) in Africa with description of a new species from the Congo. Sydowia 66: 43-54.

Heim, R. (1957). Notes preliminaries sur les agaric hallucinogènes du Mexique. Reoue de Mycologie 22: 58-104.

Heim, R. (1959). Diagnosis latines des psilocybes hallucinogènes de la stirpe Cordispora. Revue de Mycologie 24: 103-106.

Heim, R., Wasson R. G. (1958). Les champignons hallucinogènes du Mexique. Archives du muséum national d'histoire naturelle, serie 7 6: 28-42.

Index Fungorum - Authors of Fungal Names (permanentemente actualizado, consulta 2021). http://www.indexfungorum.org/names/AuthorsOfFungalNames.asp

Laessoe, T. y Petersen, J. H. (2008). Svampelivet på ækvator. Svampe 58: 1-52.

Lodge, D. J., Ammirati, F. F., O’Dell, T. E., Mueller, G. M., Huhndorf, S. M., Wang, C. J., Stokland, J. N., Schmit, J. P., Ryvarden, L., Leacock, P. R., Mata, M., Umaña L., Wu, Q. F., Czederpiltz D. (2004). Terrestrial and lignicolous macrofungi, en G. M. Mueller, G., Bills, F., Foster, M. S. (Eds.), Biodiversity of Fungi, Inventory and Monitoring Methods, pp. 127-172, San Diego: Elsevier Academic Press. https://doi.org/10.1016/B978-012509551-8/50011-8 
Ma, T., Feng, Y., Lin, X-F., Karunarathna, S. C., Ding, W-F., Hyde, K. D. (2014). Psilocybe chuxiongensis, a new bluing species from subtropical China. Phytotaxa 156 (4): 211-220. https://doi.org/10.11646/phytotaxa.156.4.3

Niveiro, N., Zuliani, P., Ramirez, N., Popoff, O., Albertó, E. (2014). Hongos agaricoides de las Yungas argentinas, Clave de géneros. Lilloa 51: 74-86.

Pegler, D. N. (1977). A preliminary agaric flora of East Africa. Kew Bulletin Additional Series 6: 1-615.

Piepenbring, M. (2008). Reportes nuevos de Agaricales para Panamá. Acta Biológica Panamensis 1: 22-38.

Ramírez Cruz, V., Guzmán, G., Guzmán-Davalos, L. (2012). New combination in the genus Deconica (Fungi, Basidiomycota, Agaricales). Sydowia 64: 217-219.

Ramírez Cruz, V., Guzmán, G., Villalobos-A, A. R., Rodríguez, A., Matheny, B., Sánchez-G, M., Guzmán-Davalos, L. (2013). Phylogenetic inference and trait evolution of the psychedelic mushroom genus Psilocybe sensu lato (Agaricales). Botany 91: 573-591. https://doi.org/10.1139/cjb-2013-0070

Schneider, C. A, Rasband, W. S., Eliceiri, K. W. (2012). NIH Image to Image J: 25 years of image analysis. Nature Methods 9: 671-675. https://doi.org/10.1038/nmeth.2089

Singer, R. (1961). Diagnoses fungorum novorum Agaricalium II. Sydowia 15: 45-83.

Singer, R. (1975). Interesting and new species of Basidiomycetes from Ecuador. Beih. Nova Hedwigia 51: 239-246.

Singer, R. (1978). Interesting and new species of Basidiomycetes from Ecuador II. Nova Hedwigia 29: 1-98.

Singer, R. (1986). The Agaricales in modern taxonomy, 4a ${ }^{\text {a }}$ Ed., Koenigstein: Koeltz Scientific Books.

Singer, R. y Smith, A. H. (1958). Mycological investigation on Teonanácatl, the mexican hallucinogenic mushroom. Part II. A texonomic monograph of Psilocybe, section Caerulescentes. Mycologia 50: 262-303. https://doi.org/10.1080/0027 5514.1958.12024726

Thiers, B. [permanentemente actualizado, consulta 2021] Index Herbariorum: a global directory of public herbaria and associated staff. New York Botanical Garden's Virtual Herbarium. Disponible en http://sweetgum.nybg.org/ih

Valencia-P, L. I. y Garzón-C, A. J. (2018). Promoción de la gestión social, cultural y económica del GAD de la parroquia rural La Esperanza del cantón Ibarra en la provincia de Imbabura. http://repositorio.utn.edu.ec/handle/123456789/8119

Van Amsterdam, J., Opperhuizen, A., Van Den Brink, W. (2011). Harm potential of magic mushroom use: a review. Regulatory Toxicology and Pharmacology 59: 423-429. https://doi.org/10.1016/j.yrtph.2011.01.006

Vellinga, E. (1988). Glossary, en C. Bas, T. H. W. Kuyper, M. E. Noordeloos \& E. C. Vellinga (Eds.), Flora Agaricina Neerlandica Vol. 1, pp. 54-64. Rotterdam: A. A. Brookfield/Balkema Publisher.

Wasson, R. G. (1957). Seeking the magic mushroom. Life 49 (19): 100-102, 109-120. https://doi.org/10.1002/j.1551-8833.1957.tb16820.x

Wright, J. E. y Albertó E. (2002). Guia de los Hongos de la región Pampeana: Hongos con Laminillas. Buenos Aires: LOLA. 